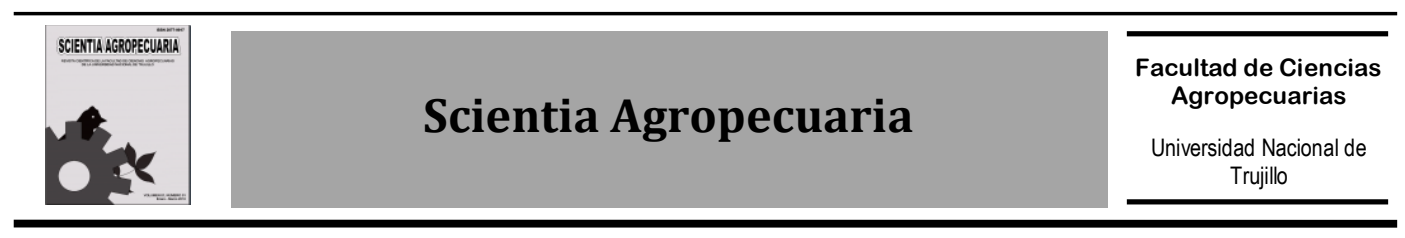

Efecto genotóxico del plaguicida fipronil en alevinos de "Gamitana" Colossoma macropomum en condiciones de laboratorio

\title{
Pesticide genotoxic effect of fipronil in alevins "Gamitana" Colossoma macropomum under laboratory conditions
}

\author{
Alberto López ${ }^{1, *}$, María Siles-Vallejos ${ }^{1}$, Emily Toscano1, Brighitte Melchor ${ }^{1}$, Guillermo Alvarez ${ }^{2}$, \\ Vidalina Heredia $^{3}$ y Violeta Norberto ${ }^{4}$ \\ ${ }^{1}$ Laboratorio de Citogenética, Facultad de Ciencias Biológicas UNMSM. \\ ${ }^{2}$ Laboratorio de Acuicultura, Facultad de Ciencias Biológicas, UNMSM. \\ ${ }^{3}$ Laboratorio de Química de los Alimentos, Facultad de Ciencias Biológicas UNMSM. \\ ${ }^{4}$ Facultad de Matemáticas, UNMSM.
}

Recibido 21 octubre 2011; aceptado 29 diciembre 2011

\begin{abstract}
Resumen
El presente trabajo evalúa la existencia de daño genotóxico en "Gamitana” ( Colossoma macropomum") al ser expuesta al plaguicida fipronil, para lo cual se utilizó el test de micronucleos (MN). Se trabajó con alevinos, dispuestos en peceras de vidrio, los cuales fueron expuestos a 3 diferentes concentraciones de fipronil (C1: $0.075 \mathrm{mg} / \mathrm{L}, \mathrm{C} 2: 0.15 \mathrm{mg} / \mathrm{L}$ y C3: $0.30 \mathrm{mg} / \mathrm{L}$ ), evaluándose a las 24 y $48 \mathrm{~h}$. Se utilizó sangre periférica para realizar el frotis. El recuento de micronúcleos y de anormalidades se realizó sobre la base de 1000 células. El índice promedio de micronúcleos indica que a $0.075 \mathrm{mg} / \mathrm{L}$ de fipronil la frecuencia promedio es el doble del control. Con respecto a las anormalidades del núcleo, se encontró una mayor frecuencia a las $48 \mathrm{~h}$ de exposición.
\end{abstract}

Palabras clave: Micronúcleos, daño nuclear, Gamitana, plaguicida.

\begin{abstract}
This study evaluates the genotoxic injury in the species Colossoma macropomum ("Gamitana") when exposed to the pesticide Fipronil, for which we used the micronucleus test (MN). We workwed with juveniles in the third stage, placed in glass tanks, which were exposed to 3 different concentrations of fipronil (C1: $0.075 \mathrm{mg} / \mathrm{L}, \mathrm{C} 2: 0.15 \mathrm{mg} / \mathrm{L}$ and C3: $0.30 \mathrm{mg} / \mathrm{L}$ ), evaluated at 24 and $48 \mathrm{~h}$. Peripheral blood was used which made the spread. The counting of micronuclei and abnormalities was made on the basis of 1000 cells. The average rate of micronuclei indicates that $0.075 \mathrm{mg} / \mathrm{L}$ the average frequency is twice the control. With regard to abnormalities of the core, was found more frequently at $48 \mathrm{~h}$ of exposure.

Keywords: Micronuclei, nuclear damage, Gamitana, pesticide.
\end{abstract}

\section{Introducción}

Las aguas continentales han sido el medio más utilizado para eliminar los productos de desecho. Hasta hace poco el poder autodepurador de los cursos de agua en la naturaleza era suficiente para restaurar y mantener el equilibrio acuático. Sin embargo las emisiones y vertidos incontrolados de naturaleza industrial y humana han sobrepasado el poder autodepurador rompiendo el ciclo hidrológico y afectando a las comunidades acuáticas. Esto ha provocado la transformación de las aguas de lagos, rios y mares en depósitos de residuos en los que el equilibrio natural está severamente perturbado $y$ en muchos casos roto (Forstner y Wittman, 1981). Muchos de estos residuos resultan ser sustancias que presentan toxicidad, la cual está referida a las propiedades específicas no deseables de la sustancia química (Beringola, 1996).

\footnotetext{
* Autor para correspondencia

Email: alopezs@unmsm.edu.pe (A. López)
} 
Una de las sustancias que puede presentar toxicidad en medios acuáticos es el fipronil, insecticida efectivo contra diversas plagas, pero con efectos contaminantes en el medioambiente y la salud humana (Carbonetto, 2004). Mulrooney et al. (1998) reporta que su persistencia es baja-moderada en el agua y en el suelo (vida media 10-130 horas en el agua y 45-530 horas en el suelo), con posterior formación de tres subproductos resultado de su degradación en el suelo RPA 20076 (amido), MB46513 (fipronildesulfinil) y RPA 104615 - y dos metabolitos importantes en el agua, incluyendo MB 45950 (sulfuro) y delsufinil, así miso señala que en condiciones aeróbicas en el suelo, se han identificado varios metabolitos, entre ellos RPA 200766 y MB 46136 (sulfona). En medioambientes acuáticos los residuos de fipronil se mueven rápidamente del agua al sedimento; más del $95 \%$ de los residuos se han encontrado en o sobre los sedimentos durante la semana en que se realiza la aplicación (Bobé et al., 1998). Existen reportes de la presencia de metabolitos del fipronil en sedimentos de río ubicados en regiones de cultivo de arroz, en Lousiana, Estados Unidos (Demcheck y Skrobialowski, 2003). Así mismo estudios metabólicos mostraron un potencial para la bioacumulación del producto fipronildesulfonil en tejidos grasos tanto en animales como humanos (Office of Prevention, Pesticides and Toxic Substances, US, 1998).

Existen algunos estudios del efecto tóxico del fipronil en especies acuáticas. Así, se han reportado estudios en "guppy" (Poecilia reticulata) encontrándose hiperexcitación a $0.1 \mathrm{mg} / \mathrm{L}$ (GómezManrique y Goncalves-Machado, 2008), en tilapias africanas (Oreochromis niloticus) se reportó que la dosis letal 50 DL (96 h) fue $42 \mu \mathrm{g} / \mathrm{L}$ (Diallo et al., 1998), asimismo es tóxico para una amplia variedad de invertebrados acuáticos (Lahr et al., 1998), asi como de alta toxicidad para los camarones y otros crustáceos, así también para las ostras (Flores 2010).

Colossoma macropomum "Gamitana" es una especie nativa de la amazonía que tiene gran demanda y sirve de alimento para las poblaciones locales. Sin embargo no hay reportes de estudios de toxicidad en dicha especie, causado por insecticidas que puedan llegar a los cursos de agua.

Un agente tóxico es aquel capaz de producir una respuesta adversa en un sistema biológico. Aquellos que producen algún tipo de alteración en el material genético o en sus componentes asociados, se les reconoce como agentes genotóxicos (Zuluaga et al., 2009).

Una forma de evaluar el daño genotóxico probable de un agroquímico sobre un organismo es mediante el Test de Micronúcleos (MN), consistente en el conteo de micronúcleos, que son cuerpos citoplasmáticos cromatínicos que se forman de fragmentos de cromosomas acéntricos o de cromosomas enteros en poblaciones celulares en división expuestas al genotóxico, como en células epiteliales o sanguíneas. Esta prueba está siendo cada vez más usada en organismos acuáticos para detectar posibles efectos mutagénicos y/o de daño nuclear (Ali et al., 2008; Normann et al., 2008).

El presente trabajo tiene por objetivo evaluar el efecto genotóxico del fipronil en alevinos de "Gamitana", utilizando el Test de micronúcleos.

\section{Materiales y métodos}

Se trabajó con alevinos de "Gamitana" (Colossoma macropomum), obtenidos a partir de reproductores procedentes de la Universidad Nacional Agraria de la Selva, dispuestos en peceras de vidrio. El volumen efectivo de las peceras fue de 1.5 L; colocadas en acuarios de $70 \mathrm{~L}$ por 24 horas, con aireación constante por medio 
de manguerillas siliconadas unidas a pipetas Pasteur. El agua utilizada para el mantenimiento de las peceras fue agua blanda, en constante aireación, a una temperatura de $30.0 \pm 0.5{ }^{\circ} \mathrm{C}$ controlada por un termostato. La alimentación fue una vez por día, con alimento extrudido, al $40 \%$ de proteína de acuerdo al peso corporal según Álvarez et al. (2008). El fotoperiodo fue de $12 \mathrm{~h}$ de luz, y $12 \mathrm{~h}$ de oscuridad. El control de los parámetros fisicoquímicos $(\mathrm{pH}, \mathrm{OD}$ y Temperatura) fue realizado una vez por semana, empleándose un multímetro para su medición.

Previo a iniciar las pruebas se dejó de alimentar a los peces por 24 horas. A cada uno de los peces se les realizó la biometría, para los cuales se obtuvo una longitud total promedio de $4.70 \pm 0.1 \mathrm{~cm}$ y una masa promedio de $2.00 \pm 0.1 \mathrm{~g}$.

Se utilizó la formulación comercial Regent $^{\circledR} 200$ SC, como fuente de fipronil (5-amino-1-[2,6-dicloro-4-(trifluorometil)phenil]-4[(trifluorometil)-sulfinil]-Hpirazol -3-carbonitril). La solución stock y las concentraciones fueron preparadas 1 hora antes de iniciar la prueba.

Se trabajó con tres concentraciones $(\mathrm{C} 1$ : $0.075 \mathrm{mg} / \mathrm{L}, \quad$ C2: $0.15 \mathrm{mg} / \mathrm{L}$ y C3: 0.30 $\mathrm{mg} / \mathrm{L})$, teniendo un control, y una solución stock (1ml del fipronil en $5 \mathrm{~L}$ de agua blanda). Para cada concentración se utilizaron dos peces, y se realizaron dos réplicas por concentración, evaluándose un grupo a las $24 \mathrm{~h}$ y otro a las $48 \mathrm{~h}$.

La sangre periférica se obtuvo mediante corte en la región posterior al opérculo, previamente se colocaron a los peces en agua fría a una temperatura de $4^{\circ} \mathrm{C}$ durante 5 minutos, luego se desinfectó el tejido epitelial con alcohol al 70\%. Se realizó el frotis en láminas, dejándose secar a temperatura ambiente por 30 minutos para luego ser fijadas en solución etanol: ácido acético $(3: 1 \mathrm{v} / \mathrm{v})$ por una hora y finalmente ser teñidas con Giemsa al 2\% durante una hora. Se realizó el conteo de micronúcleos (MN) y anormalidades sobre la base de 1000 células, utilizándose un microscopio compuesto con cámara digital incorporada; el aumento de lectura fue de 1000X. La identificación de anormalidades se realizó según lo descrito por Jirangkoorkhul (2007). Las frecuencias de micronúcleos y anormalidades se expresan por 1000 células (\%o).

Se calculó el índice de proliferación (PI) en base a la frecuencia de las células que presentan micronúcleo (MNC \%o) del control negativo respecto al MNC \%o calculado de las muestras, de acuerdo a la siguiente fórmula (Palma, 2005):

\section{$P I=M N C \% / M N C \%$ del control negativo}

Los Criterios de Identificación de Micronúcleos fueron de acuerdo también a lo señalado por Palma (2005). Se aplicó en el análisis estadístico el descriptor mediana como indicador del grado de dispersión de las deformaciones y micronúcleos. Para el procesamiento de los datos se utilizó el software Excel 2007 y SPSS versión 15 .

\section{Resultados y discusión}

Se observaron eritrocitos con tres tipos de núcleo: aquellos que presentaban un núcleo uniformemente teñido de forma ovalada y compacta (Figura 1), otros que presentaban pequeños cuerpos nucleares extras, catalogados como micronúcleos, y los que presentaban deformaciones en el núcleo (Figura 2).

Las frecuencias encontradas para micronúcleos y el índice PI se muestran en las Tablas 1 y 2 . Estos resultados revelan que hay presencia de micronúcleos a las 48 h de exposición (Figura 2) y el índice promedio de micronucleos indica que a la concentración de $0.075 \mathrm{mg} / \mathrm{L}$ la frecuencia promedio es el doble del control. 


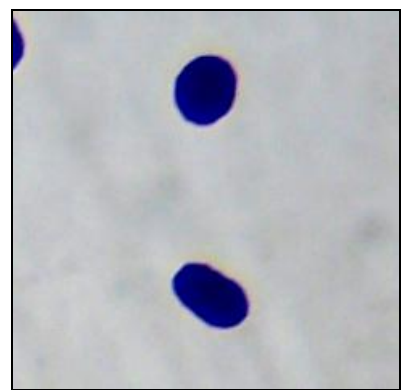

Figura 1. Fotomicrografía de núcleos normales de eritrocitos de Gamitana. Aumento 1000X.

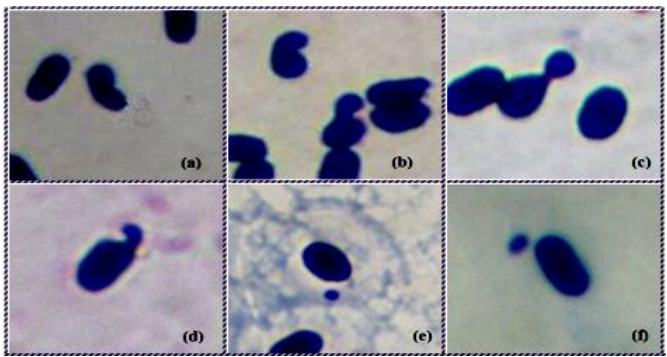

Figura 2. Fotomicrografías de Eritrocitos de Colossoma macropomum con Anormalidades nucleares (a) Núcleo Lobado, (b) Núcleo en Muesca, (c) y (d) Núcleo Globulado, (e) y (f) Micronúcleos. Aumento 1000X.

\section{Tabla 1}

Frecuencia de micronúcleos (\%) en eritrocitos de sangre periférica de Colossoma macropomum expuestos a fipronil.

\begin{tabular}{lcc}
\hline $\begin{array}{l}\text { Concentración } \\
\text { de fipronil }(\mathrm{mg} / \mathrm{L})\end{array}$ & $\begin{array}{c}\text { MN\%o } \\
24 \mathrm{~h}\end{array}$ & $\begin{array}{c}\mathrm{MN} \% \text { o } \\
48 \mathrm{~h}\end{array}$ \\
\hline 0.075 & 0 & 0.119 \\
0.15 & 0 & 0.065 \\
0.30 & 0 & 0.063 \\
\hline control & 0.033 & 0.024 \\
\hline
\end{tabular}

\section{Tabla 2}

Relación de Frecuencias (PI) de micronucleos en eritrocitos de sangre periférica de Colossoma macropomum expuestos a fipronil.

\begin{tabular}{lcc}
\hline $\begin{array}{l}\text { Concentración } \\
(\mathrm{mg} / \mathrm{L})\end{array}$ & $\begin{array}{c}\text { Promedio } \\
\text { de MN \% }\end{array}$ & PI \\
\hline 0.075 & 0.060 & 2.069 \\
0.15 & 0.033 & 1.138 \\
0.30 & 0.032 & 1.103 \\
\hline control & 0.029 & - \\
\hline
\end{tabular}

Con respecto a las anormalidades del núcleo, se diferenciaron tres tipos: globulado, lobado y en muesca (Figura 2); encontrándose que se presentan con mayor frecuencia a las 48 horas de exposición, siendo las de núcleo globulado y en muesca las de mayor presencia (Tabla 3 ).

\section{Tabla 3}

Frecuencia de anormalidades nucleares en eritrocitos de sangre periférica de Colossoma macropomum expuestos a fipronil.

\begin{tabular}{llcc}
\hline $\begin{array}{l}\text { Concentración } \\
(\mathrm{mg} / \mathrm{L})\end{array}$ & \multicolumn{1}{c}{ Tipo } \\
& Anormalidad & $\begin{array}{c}\text { Frecuencia } \\
\% 0 \\
24 \mathrm{~h}\end{array}$ & $\begin{array}{c}\text { Frecuencia } \\
\% 0 \\
48 \mathrm{~h}\end{array}$ \\
\hline \multirow{3}{*}{0.075} & Globulado & 0.109 & 0.053 \\
& Lobado & 0.036 & 0.026 \\
& En muesca & 0.055 & 0.225 \\
\hline \multirow{3}{*}{0.15} & Globulado & 0.036 & 0.130 \\
& Lobado & 0 & 0 \\
& En muesca & 0.055 & 0.022 \\
\hline \multirow{3}{*}{0.30} & Globulado & 0.023 & 0.188 \\
& Lobado & 0 & 0.038 \\
& En muesca & 0.047 & 0.088 \\
\hline \multirow{2}{*}{ Control } & Globulado & 0.056 & 0.024 \\
& Lobado & 0.011 & 0 \\
& En muesca & 0 & 0.047 \\
\hline
\end{tabular}

En las figuras 3 y 4 se representan las medianas de las deformaciones encontradas para cada concentración, a las 24 y 48 $\mathrm{h}$ de exposición respectivamente. En la concentración de $0.075 \mathrm{mg} / \mathrm{L}$ se obtuvo, en promedio, 5.5 deformaciones, que es la más alta con respecto a las otras concentraciones y también tiene mayor dispersión (Figura 3). Para las 48 h, se observa que el mayor número promedio es de 12.5 deformaciones para la concentración a $0.3 \mathrm{mg} / \mathrm{L}$, siendo la mediana (12.5) ligeramente mayor que la observada para la concentración de $0.075 \mathrm{mg} / \mathrm{L}$ (mediana igual a 11.5), existiendo, además, mayor dispersión para una concentración de $0.3 \mathrm{mg} / \mathrm{L}$ (Figura 4).

Los MN son masas de cromatina que se evidencian como pequeños núcleos, los cuales surgen a partir de fragmentos de cromosomas o cromosomas enteros intactos que quedaron retrasados en la etapa de anafase de células en división (Jirangkoorkhul y Sahaphong, 2007). 


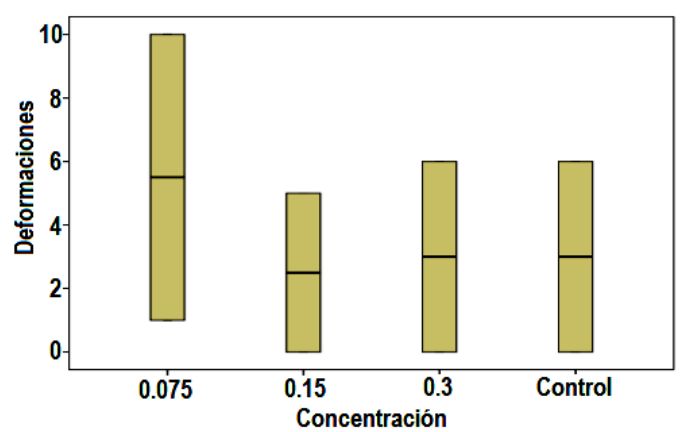

Figura 3. Medianas de las deformaciones nucleares vs. Concentraciones de fipronil (mg/L) a 24 horas de exposición.

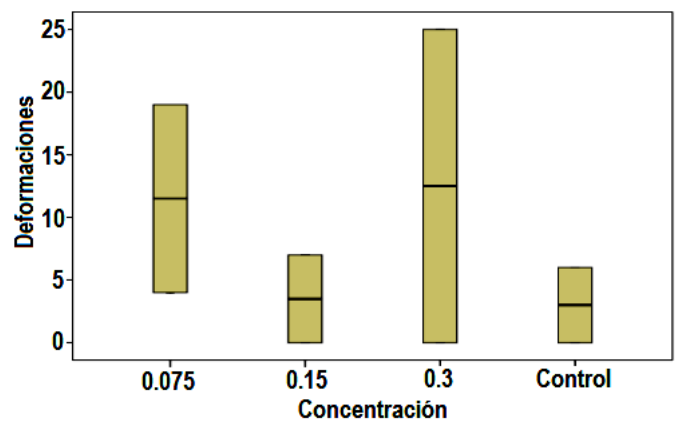

Figura 4. Medianas de las deformaciones nucleares vs. Concentraciones de fipronil (mg/L) a 48 horas de exposición.

El test de micronúcleos aplicado a biomonitoreo genotóxico de contaminantes resulta una herramienta práctica en mutagénesis ambiental (Palma, 2005). AISabti y Hardig (1990) reporta que los organismos acuáticos como los peces son excelentes biomonitores para detectar contaminantes que se encuentran en diferentes estratos acuáticos, mediante la evaluación de micronúcleos. Nuestros resultados, en Gamitana, muestran la presencia de micronúcleos, los cuales se presentan en mayor frecuencia a una concentración de $0.075 \mathrm{mg} / \mathrm{L}$ (Tabla 1) lo que estaría indicando que el fipronil habría causado la formación de estos fragmentos cromosómicos. La presencia de micronúcleos en el control, se habría dado espontánea-mente, lo que concuerda con lo reportado en pruebas de genotoxicidad para otras especies de peces (Oliveria et al., 2004; Prieto et al., 2007). El mecanis- mo mediante el cual pueden formarse micro-núcleos espontáneamente es por perdida mitótica de fragmentos acéntricos, donde cualquier fragmento cromosómico que no posea centrómero no podrá integrarse a un núcleo, durante la división celular, pues carece del elemento que le permita orientarse en el huso acromático. Estos elementos quedan rezagados y permanecerán en el citoplasma conformando uno o varios núcleos muy pequeños, que son los denominados micronúcleos (Heddle et al., 1991; Martínez, 2005).

En altas concentraciones de fipronil observamos una disminución de micronúcleos. Según Dailianis et al. (2003), citado por Sotil et al. (2007), aparentemente los incrementos en la concentración y tiempos de exposición a agentes mutagénicos inhibirían la actividad mitótica. Además Prieto et al. (2008) mencionan que las disminuciones de $\mathrm{MN}$ después de una fase de incremento, están relacionadas con el tipo de genotóxico, la concentración utilizada, la forma de administración y la respuesta genética que tenga cada especie.

Con respecto a las anormalidades del núcleo, se diferenciaron tres tipos de deformaciones: globulado, lobado y en muesca (Figura 2); encontrándose que se presentan con mayor frecuencia a las 48 horas de exposición, siendo las de núcleo globulado y en muesca las de mayor presencia (Tabla 3). Los núcleos ovalados y uniformes corresponden a núcleos de aspecto normal, tal como se han reportado en otras especies de peces (Valenzuela, 2003; Silveira et al., 2005).

Si bien es cierto que se puede evidenciar el daño de un tóxico mediante la frecuencia de MN, no siempre ocurrirá de esta forma, ya que el tóxico no necesariamente va a causar una ruptura del ADN sino también puede provocar diferentes anormalidades nucleares como las reportadas por Jirangkoorkul y Sahaphong (2007) como 
núcleo bilobulado, en muesca, globulado y lobado. En este trabajo se reportan anormalidades nucleares en un orden de frecuencia de globulado, lobado y en muesca, que son tres de las cuatro anormalidades mencionadas.

\section{Conclusiones}

Los resultados obtenidos para alevinos de Colossoma macropomun nos indican que el fipronil, a la concentración de 0.075 $\mathrm{mg} / \mathrm{L}$ ha ocasionado la formación de micronucleos, disminuyendo la frecuencia de los mismos a las concentraciones de $0.15 \mathrm{mg} / \mathrm{L}$ y $0.30 \mathrm{mg} / \mathrm{L}$. Sin embargo, las anormalidades nucleares sí están presentes en las tres concentraciones utilizadas, siendo la de núcleo globulado la que mayor frecuencia reporta. Esto indica que el fipronil actúa como un agente que ocasiona anormalidades nucleares en los eritrocitos de Colossoma macropomun, pero no así causa un mayor efecto en la formación de micronúcleos.

\section{Agradecimientos}

Al Consejo Superior de Investigaciones de la UNMSM que permitió la realización del presente trabajo como parte del proyecto 091001231. Así también a Miguel Saldaña por su valiosa colaboración en el mantenimiento de los peces.

\section{Referencias}

AI-Sabti K.: Hardig, J. 1990. Micronucleus monitoring the genotoxic effects of the industrial waste products in the Baltic sea, Sweden. Comp. Bioch. Physiol. 97C: 179-182.

Ali FK.; El-Shehawi, A.; Seehy, M. 2008. Micronucleus test in fish genome: A sensitive monitor for aquatic pollution. African journal of biotechnology 7(5): 606612.

Alvarez, P.; Ortega, R.; Vela, M.; Canales, D.; Saldaña, M 2008. Efecto de la temperatura y densidad sobre el peso y longitud estándar final de alevines de "gamitana" Colossoma macropomum con alimento extruido y peletizado. Instituto de Ciencias Biológicas Antonio Raimondi. Universidad Nacional Mayor de San Marcos. Lima, Perú.
Beringola L. 1996. Genotoxicidad de las aguas del río Tajo. Tesis para Doctorado. Facultad de Ciencias Biológicas. U. Complutense de Madrid. <http://eprints. ucm.es/tesis/19911996/X/3/X3040101.pdf>.

Bobé A.; Cooper, J.; Coste, C.; Muller, M. 1998. Behaviour of fipronil in soil under Sahelian Plain field conditions. Pestic. Sci. 52(3): 275-281.

Carbonetto, G. 2004. Fipronil. Pesticides News. 48(1): 20.

Demcheck, D. K.; Skrobialowski, S. C. 2003. Fipronil and degradation products in the rice-producing areas of the Mermantau river basin, Louisiana, february-september 2000. Fact Sheet FS-010-03. National Water Quality Assessment Program. U.S.G.S. Baton Rouge, LA.

Diallo A., Diagne,M.; Ndour, K.; Lahr, J. 1998. Laboratory toxicity tests with eight acridicides on Oreochromis niloticus (Pices, Cichlidae). Environmental side-effects of locust and grasshopper control 3: 188-204.

Flores, R. 2010. Evaluación ambiental del fipronil. Tesis para optar al Grado de Maestría en Ciencias Ambientales. Facultades de Ciencias Químicas, Ingeniería y Medicina. Universidad Autónoma de San Luis de Potosí. México.

Forstner, U.; Wittman, G. 1981. Metal pollution in the aquatic environment. $2^{\mathrm{a}}$ edición. Berlín: SpringerVerlag.

Gómez-Manrique, W.; Goncalves-Machado, N. 2008. Toxicidad aguda y riesgo ambiental del fipronil para Guppy (Poecilia reticulata). Biologist 6(2): 85-93.

Heddle, J.; Cimino, M.; Hayashi, M.; Romagna, F.; Shelby, M.; Tucker, J.; McGregor, J. 1991. Micronuclei as an index of cytogetic damage: past, present and future. Environ. Mol. Mutag. 18: 277-291.

Jiraungkoorskul, W.; Sahaphong, S. 2007. Efficacy of ascorbic acid reducing waterborne copper toxicity in butterfish (Poronottus triacanthus). Journal of Biological Sciences 7(4): 620-625.

Lahr A.; Badji, A.; Ndour, K.; Diallo, D. 1998. Acute toxicity tests with Streptocephalus sudanicus (Branchipoda, Anostraca) and Anisops sardeus (Hemiptera, Notonectidae) using insecticides for Desert Locust control. Environmental side-effects of locust and grasshopper control 3: 39-57.

Martínez S. 2005. El cerdo joven como bioindicador de concentraciones bajas de genotóxicos mediante la prueba de micronúcleos en eritrocitos de sangre periférica. Tesis para obtener el grado de doctor en ciencias Médicas. Facultad de Ciencias Médicas. Universidad de Colima, México.

Mulrooney J.; Wolfenbarger, D.; Howard, K.; Goli, D.. 1998. Efficacy of ultra low volume and high volume applications of fipronil against the boll weevil. Journal of Cotton Science. 2(3): 110-116.

Normann C.A.; Fonseca, J.C.; Veiga, V. 2008. Micronuclei in red blood cells of armored catfish Hypostomus plecotomus exposed to potassium dichromate. African Journal of Biotechnology 7(7): 893-896.

Office of Prevention, Pesticides and Toxic Substances, US. 1998. Fipronil for use on Rice (Regent, Icon) and Pets (Frontline). HED Risk Assessment. USEPA. Washington DC. 


\section{A. López et al. / Scientia Agropecuaria 2(2011) 247 - 253}

Oliveria A.; Ramírez, B.; Prieto, F.; Galán, C.. 2004. Bioacumulación y daños genotóxicos en Pez Cebra (Danio rerio) por arsénico en aguas de Zimapán, Hidalgo (México). Ensayos en cortos plazos. Revista AquaTIC. 21: 62-70.

Palma, P. 2005. Ensayo de micronúcleos en eritrocitos de Oncorhynchus mykiss como herramienta para evaluar la exposición a pesticidas potencialmente genotóxicos en el río Traiguen. Tesis para optar al Grado de Licenciado en Recursos Naturales. Facultad de Ciencias. Universidad Católica de Temuco. Chile.

Prieto, F.; Ramírez, B.; Scout, W.; Gaytan, J.C.; Zuñiga, A. 2007. Toxicidad y teratogenésis por arsénico en aguas en el pez cebra. Rev. Toxicol. 24: $18-22$.

Prieto, Z.; León, J.; Quijano, C.; Fernández, R.; Polo, E.; Vallejo, R.; Villegas, L. 2008. Efecto genotóxico del dicromato de potasio en eritrocitos de sangre periférica de Oreochromis niloticus (tilapia). Revista Peruana de Medicina Experimental y Salud Pública 25(1): 51-58.
Silveira R.; Cruz, Y.; Martínez, M. 2005. Características morfológicas y citoquímicas de las células de la sangre periférica de Oreochromis aureus S. Cichlidae. Revista Electrónica de Veterinaria REDVET 6(10):1-8 <http://www.veterinaria.org/revistas/redvet/n101005.ht $\mathrm{ml}>$.

Sotil, G.; Alvis, R.; Francia, J.; Shiga, B. 2007. Aplicación de dos biomarcadores para el análisis de lesiones en el DNA de bivalvos marinos. Rev. Peru. Biol. Número especial 13(3): 249-253.

Valenzuela A.; Oyarzún, C.; Silva, V. 2003. Células sanguíneas de Schroederichthys chilensis (Quichenot 1848) (Elasmobranchii, scyliorhinidae): la serie blanca. Gayana 67(1): 130-136.

Zuluaga, M.; Valencia, A.; Ortiz, I. 2009. Efecto genotóxico y mutagénico de contaminantes atmosféricos. Medicina Universidad Pontificia Bolivariana UPB. 28(1): 33-41. 